\title{
Eosin Y Catalyzed Visible-light-promoted One -Pot Facile Synthesis of 1,3,4- Thiadiazole
}

\author{
Vishal Srivastava, ${ }^{\mathrm{a}}$ Pravin K. Singh, ${ }^{\mathrm{b}}$ and Praveen P. Singh ${ }^{\mathrm{c}, *}$ \\ ${ }^{a}$ Laboratory of Green Synthesis, Department of Chemistry, University of Allahabad, Allahabad - 211002, India \\ ${ }^{\mathrm{b}}$ Food Analysis and Research Lab, Centre of Food Technology, University of Allahabad, Allahabad - 211002, India \\ ${ }^{\mathrm{c}}$ Department of Chemistry, United College of Engineering \& Research, Naini, Allahabad - 211010, India
}

RECEIVED JULY 23, 2014; REVISED DECEMBER 26, 2014; ACCEPTED JANUARY 9, 2015

Abstract. A novel one-pot visible light irradiated synthesis of 1,3,4-thiadiazole from aldehydes and thioacyl hydrazides have been reported in presence of eosin $\mathrm{Y}$ as an organophotoredox catalyst at room temperature under aerobic condition. This synthesis includes application of air and visible light as inexpensive, readily available, non-toxic and sustainable regents, which fulfils the basic principle of green chemistry.

Keywords: Eosin Y, visible-light, organophotoredox, green chemistry, aerobic condition

\section{INTRODUCTION}

Sunlight is a unique and renewable natural source. ${ }^{1}$ The development of methods to efficiently harness the solar radiation energy has emerged as one of the central scientific challenges of the twenty first century. ${ }^{2-3}$ Therefore, some pioneering researchers have dedicated to converting solar energy into chemical energy for chemical transformations. ${ }^{4-5}$ Recently, a surge of interest from the synthetic community has brought photoredox manifolds to the forefront of catalysis. In this sequence visible light photoredox catalysis has recently received much attention in organic synthesis owing to ready availability, sustainability, non-toxicity and ease of handling of visible light. ${ }^{6-11}$ In their revolutionary work in this area, MacMillan, ${ }^{12}$ Yoon $^{13}$ and Stephenson ${ }^{14-15}$ have used Ruthenium and Iridium complexes as the photoredox catalyst, which has inspired the development of several powerful methods for various chemical transformations useful in organic synthesis. ${ }^{16}$

However, these transition metal based photocatalysts disadvantageously exhibit high cost, low sustainability and potential toxicity. Recently, a superior alternative to transition metal photoredox catalysts, especially metal-free organic dyes such as eosin Y, fluorescein, Rose Bengal, nile red, perylene and rhodamine $\mathrm{B}$ have been used as economically and ecologically superior surrogates for $\mathrm{Ru}(\mathrm{II})$ and $\mathrm{Ir}(\mathrm{II})$ complexes in visible-light promoted organic transformations involv- ing $\mathrm{SET}^{17-20}$ (single electron transfer). These organic dyes have got much more attention with the last few years also due to easy handling, eco-friendly and have great potential for applications in visible-light-mediated organic synthesis, ${ }^{21-24}$ which fulfils the basic principle of green chemistry.

The five-membered heterocyclic compounds have a great application and importance in heterocyclic chemistry. Among them thiadiazole is a versatile moiety that exhibits a wide variety of biological activities. Thiadiazole moiety acts as "hydrogen binding domain" and "two-electron donor system". It also acts as a constrained pharmacophore. Many drugs containing thiadiazole nucleus are available in the market such as acetazolamide, methazolamide, sulfamethazole, etc. 1,3,4thiadiazoles are associated with diverse pharmacological activities such as analgesic, ${ }^{25}$ antidepressant, anxiolytic, ${ }^{26}$ anticonvulsant, ${ }^{27-28}$ anti-inflammatory, ${ }^{29}$ antimicrobial, ${ }^{30}$ anti-tubercular, ${ }^{31}$ antitumor, ${ }^{32}$ and anti-viral activities. $^{33}$

The synthesis of 1,3,4-thiadiazole have not been reported by photooxidation reaction so far. Meanwhile the aerobic oxygen has received a great importance in research during present time. ${ }^{34-35}$ In general, organosulfur/nitrogen compounds have been frequently used as precursors in radical reactions because they form radicals very readily. ${ }^{36-38}$ Encouraged by organocatalytic visible-light-mediated aerobic oxidative transformations ${ }^{39-40}$ and in continuation of our work on develop-

\footnotetext{
* Author to whom correspondence should be addressed. (E-mail: ppsingh23@gmail.com)
} 
<smiles>[R]C=NNC([R])=S</smiles>

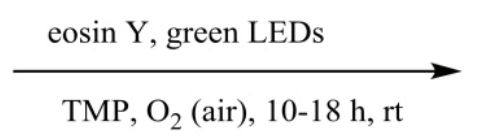<smiles>[R]c1nnc([R])s1</smiles>

Scheme 1. Eosin Y catalysed visible light promoted synthesis of 1,3,4-thiadiazole.

ment of novel environmentally benign synthesis ${ }^{41-44}$ herein we report a simple, visible light irradiated, efficient and green protocol for the synthesis of 1,3,4thiadiazoles, using eosin $\mathrm{Y}$ as photocatalyst with excellent yield as depicted in Scheme 1.

\section{EXPERIMENTAL}

Melting points were determined by open glass capillary method and are uncorrected. All chemicals used were reagent grade and were used as received. ${ }^{1} \mathrm{H}$ NMR and ${ }^{13} \mathrm{C}$ NMR spectra were recorded on a Bruker AVANCE DPX $(400 \mathrm{MHz}$ and $75 \mathrm{MHz}) \mathrm{FT}$ spectrometer in DMSO using TMS as an internal reference (chemical shift in $\delta, \mathrm{ppm}$ ). Mass spectra were recorded on JEOL SX-303 (FAB) mass spectrophotometer at 70ev. Elemental analyses were carried out using a Coleman automatic $\mathrm{C}, \mathrm{H}, \mathrm{N}$ analyser.

\section{General Procedure for the Synthesis of 2,5-disubsti- tuted 1,3,4-thiadiazole 4(a-n):}

A solution of an aldehyde $\mathbf{1}(\mathbf{a}-\mathbf{n})(1.0 \mathrm{mmol})$ and an thioacylhydrazide $\mathbf{2}(\mathbf{a}-\mathbf{n})(1.0 \mathrm{mmol})$ in $\mathrm{MeCN}(3 \mathrm{~mL})$ was heated at $65^{\circ} \mathrm{C}$ for $2-6 \mathrm{~h}$ to form the corresponding thioacylhydrazone (as monitored by TLC). Then, eosin $\mathrm{Y}(2.0 \mathrm{~mol} \%)$ and TMP (2.0 equiv.) were added and the mixture was irradiated with green LEDs $(2.4 \mathrm{~W}, 120$ $1 \mathrm{~m}$ ) with stirring under an air atmosphere at rt for 10-18 h. After completion of the reaction (monitored by TLC), water $(5 \mathrm{~mL})$ was added and the mixture was extracted with EtOAc $(3 \times 5 \mathrm{~mL})$. The combined organic phase was dried over $\mathrm{MgSO}_{4}$, filtered and evaporated under reduced pressure. The resulting product was purified by silica gel column chromatography using a gradient mixture of hexane/ethyl acetate as eluent to afford an analytically pure sample of $\mathbf{4}(\mathbf{a}-\mathbf{n})$. All the products are known compounds and were characterized by the comparison of their spectral data with those reported in the literature. In the ${ }^{1} \mathrm{H}$ NMR spectra, signal of respective protons of newly synthesized compounds $\mathbf{4}(\mathbf{a}-\mathbf{n})$ showed the peaks for $-\mathrm{CH}_{3},-\mathrm{OCH}_{3}$ and aromatic protons.

4a. 2-(4-chlorophenyl)-5-phenyl-1,3,4-thiadiazole m.p. $180{ }^{\circ} \mathrm{C}, m / z$ : 272.02; Mol. Wt: $272.75 ;{ }^{1} \mathrm{H}$ NMR (400 MHz, DMSO-d d $_{6} \delta /$ ppm: 7.41-7.60 (m, 5H, ArH),
7.65-7.74 (d, 2H, $\left.J=8.1 \mathrm{~Hz}, 2^{\prime}, 6^{\prime}-\mathrm{ArH}\right), 8.02-8.15(\mathrm{~d}$, $\left.2 \mathrm{H}, J=8.4 \mathrm{~Hz}, 3^{\prime}, 5^{\prime}-\mathrm{ArH}\right),{ }^{13} \mathrm{C} \mathrm{NMR}(75 \mathrm{MHz}$, DMSO-d ${ }_{6}$ ) $\delta / \mathrm{ppm}: 128.7,128.9,129.2,129.3,130.9$, 131.6, 133.5, 134.3, 174.1; Anal. Calcd for $\mathrm{C}_{14} \mathrm{H}_{9} \mathrm{ClN}_{2} \mathrm{~S}$ : C, 61.65; H, 3.33; N, 10.27. Found: C, 61.42; H, 3.31; N, 10.25 .

4b. 2-(3-chlorophenyl)-5-phenyl-1,3,4-thiadiazole m.p. $187{ }^{\circ} \mathrm{C}, \mathrm{m} / z$ : 272.02; Mol. Wt: 272.75; ${ }^{1} \mathrm{H}$ NMR (400 MHz, DMSO-d $\left.{ }_{6}\right) \delta /$ ppm: 7.41-7.60 (m, 5H, ArH), 7.65-7.74 (dd, $\left.1 \mathrm{H}, J=8.1 \mathrm{~Hz}, J=8.9 \mathrm{~Hz}, 5^{\prime}-\mathrm{ArH}\right)$, $7.80-7.85\left(\mathrm{~d}, 1 \mathrm{H}, J=8.1 \mathrm{~Hz}, 4^{\prime}-\mathrm{ArH}\right), 7.91-8.00(\mathrm{~d}$, $\left.1 \mathrm{H}, J=8.3 \mathrm{~Hz}, 6^{\prime}-\mathrm{ArH}\right), 8.04-8.13$ (s, $\left.1 \mathrm{H}, 2^{\prime}-\mathrm{ArH}\right),{ }^{13} \mathrm{C}$ NMR (75 MHz, DMSO-d $\left.{ }_{6}\right) \delta /$ ppm: 127.4, 128.7, 128.8, 129.0, 129.2, 130.9, 133.5, 134.8, 134.9, 174.1; Anal. Calcd for $\mathrm{C}_{14} \mathrm{H}_{9} \mathrm{ClN}_{2} \mathrm{~S}$ : C, 61.65; H, 3.33; N, 10.27. Found: C, 61.42; H, 3.31; N, 10.25.

4c. 2-(2-chlorophenyl)-5-phenyl-1,3,4-thiadiazole m.p. $195{ }^{\circ} \mathrm{C}, \mathrm{m} / z$ : 272.02; Mol. Wt: $272.75 ;{ }^{1} \mathrm{H}$ NMR (400 MHz, DMSO-d 6 ) $\delta /$ ppm: 7.41-7.60 (m, 7H, ArH, 4', 5'- ArH), 7.65-7.70 (d, 1H, $\left.J=8.1 \mathrm{~Hz}, 3^{\prime}-\mathrm{ArH}\right)$, $7.73-7.78\left(\mathrm{~d}, 1 \mathrm{H}, J=8.1 \mathrm{~Hz}, 66^{\prime}-\mathrm{ArH}\right),{ }^{13} \mathrm{C} \mathrm{NMR}(75$ MHz, DMSO-d 6 ) $\delta /$ ppm: 127.3, 128.7, 128.9, 129.2, 129.3, 130.1, 130.9, 132.2, 133.5, 136.9, 174.1; Anal. Calcd for $\mathrm{C}_{14} \mathrm{H}_{9} \mathrm{ClN}_{2} \mathrm{~S}$ : C, 61.65; H, 3.33; N, 10.27 . Found: C, 61.42; H, 3.31; N, 10.25.

4d. 2-(4-methoxyphenyl)-5-phenyl-1,3,4-thiadiazole m.p. $215{ }^{\circ} \mathrm{C}, m / z$ : 268.07; Mol. Wt: 268.33; ${ }^{1} \mathrm{H}$ NMR (400 MHz, DMSO-d 6 ) $\delta / \mathrm{ppm}: 3.83\left(\mathrm{~s}, 3 \mathrm{H},-\mathrm{OCH}_{3}\right)$, 7.41-7.50 (m, 5H, ArH), 7.58-7.69 (d, 2H, $J=8.1 \mathrm{~Hz}$, 3', 5'-ArH), 8.05-8.13 (d, 2H, $\left.J=8.4 \mathrm{~Hz}, 2^{\prime}, 6^{\prime}-\mathrm{ArH}\right)$, ${ }^{13} \mathrm{C}$ NMR $\left(75 \mathrm{MHz}\right.$, DMSO-d $\left.\mathrm{d}_{6}\right) \delta / \mathrm{ppm}: 55.8,114.8$, 125.8, 128.5, 128.7, 129.2, 130.9, 133.5, 160.6, 174.1; Anal. Calcd for $\mathrm{C}_{15} \mathrm{H}_{12} \mathrm{~N}_{2}$ OS: C, 67.14; H, 4.51; N, 10.44. Found: C, 67.12; H, 4.50; N, 10.40 .

\section{4e. 2,5-diphenyl-1,3,4-thiadiazole}

m.p. $140{ }^{\circ} \mathrm{C}, m / z$ : 238.06; Mol. Wt: $238.31 ;{ }^{1} \mathrm{H}$ NMR (400 MHz, DMSO-d 6 ) $\delta /$ ppm: 7.41-8.03 (m, 10H, ArH), ${ }^{13} \mathrm{C}$ NMR (75 MHz, DMSO-d 6 ) $\delta /$ ppm: 128.7, 129.2, 130.9, 133.5, 174.1; Anal. Calcd for $\mathrm{C}_{14} \mathrm{H}_{10} \mathrm{~N}_{2} \mathrm{~S}$ : C, 70.56; H, 4.23; N, 11.76. Found: C, 67.12; H, 4.50; N, 10.40 .

4f. 2-(4-bromophenyl)-5-phenyl-1,3,4-thiadiazole m.p. $250{ }^{\circ} \mathrm{C}, \mathrm{m} / \mathrm{z}$ : 317.96; Mol. Wt: $317.20 ;{ }^{1} \mathrm{H}$ NMR (400 MHz, DMSO-d $\mathrm{d}_{6}$ ) $\delta / \mathrm{ppm}: 7.41-7.60$ (m, 5H, ArH), 
$7.63-7.84$ (d, 2H, $\left.J=8.2 \mathrm{~Hz}, 2^{\prime}, 6^{\prime}-\mathrm{ArH}\right), 8.00-8.13$ (d, $\left.2 \mathrm{H}, J=8.5 \mathrm{~Hz}, 3^{\prime}, 5^{\prime}-\mathrm{ArH}\right),{ }^{13} \mathrm{C} \mathrm{NMR}(75 \mathrm{MHz}$, DMSO-d ${ }_{6}$ ) $\delta /$ ppm: 123.1, 128.7, 129.2, 129.7, 130.9, 132.1, 132.5, 133.5, 174.1; Anal. Calcd for $\mathrm{C}_{14} \mathrm{H}_{9} \mathrm{BrN}_{2} \mathrm{~S}$ : C, 53.01; H, 2.86; N, 8.83. Found: C, 52.99; H, 2.84; N, 8.81.

4g. 2-(3-bromophenyl)-5-phenyl-1,3,4-thiadiazole m.p. $258{ }^{\circ} \mathrm{C}, m / z$ : 317.96; Mol. Wt: 317.20; ${ }^{1} \mathrm{H}$ NMR (400 MHz, DMSO-d 6 ) $\delta /$ ppm: 7.41-7.60 (m, 5H, ArH), $7.67-7.78\left(\mathrm{~d}, 1 \mathrm{H}, J=8.2 \mathrm{~Hz}, J=8.7 \mathrm{~Hz}, 5^{\prime}-\mathrm{ArH}\right)$, 7.81-7.89 (d, $\left.1 \mathrm{H}, J=8.3 \mathrm{~Hz}, 4^{\prime}-\mathrm{ArH}\right), 7.90-8.02$ (d, $\left.1 \mathrm{H}, J=8.1 \mathrm{~Hz}, 6^{\prime}-\mathrm{ArH}\right), 8.04-8.14$ (s, $\left.1 \mathrm{H}, 2^{\prime}-\mathrm{ArH}\right),{ }^{13} \mathrm{C}$ NMR (75 MHz, DMSO-d $\left.{ }_{6}\right) \delta /$ ppm: 122.2, 128.1, 128.7, 129.2, 129.9, 130.9, 131.4, 133.1, 133.5, 135.7, 174.1; Anal. Calcd for $\mathrm{C}_{14} \mathrm{H}_{9} \mathrm{BrN}_{2} \mathrm{~S}$ : C, 53.01; H, 2.86; N, 8.83. Found: C, 52.99; H, 2.84; N, 8.81.

4h. 2-(4-nitrophenyl)-5-phenyl-1,3,4-thiadiazole m.p. $230{ }^{\circ} \mathrm{C}, m / z$ : 283.04; Mol. Wt: $283.31 ;{ }^{1} \mathrm{H}$ NMR (400 MHz, DMSO-d 6 ) $\delta /$ ppm: 7.41-7.60 (m, 5H, ArH), 7.70-7.94 (d, 2H, $\left.J=8.1 \mathrm{~Hz}, 2^{\prime}, 6^{\prime}-\mathrm{ArH}\right), 8.01-8.18$ (d, $\left.2 \mathrm{H}, J=8.3 \mathrm{~Hz}, 3^{\prime}, 5^{\prime}-\mathrm{ArH}\right),{ }^{13} \mathrm{C} \mathrm{NMR}(75 \mathrm{MHz}$, DMSO-d ${ }_{6}$ ) $\delta /$ ppm: $124.4,128.4,128.7,129.2,130.9$, 133.5, 139.6, 147.9, 174.1; Anal. Calcd for $\mathrm{C}_{14} \mathrm{H}_{9} \mathrm{~N}_{3} \mathrm{O}_{2} \mathrm{~S}$ : C, 59.35; H, 3.20; N, 14.83. Found: C, 59.31; H, 3.18; N, 14.81 .

4i. 2-(3-nitrophenyl)-5-phenyl-1,3,4-thiadiazole m.p. $242{ }^{\circ} \mathrm{C}, m / z$ : 283.04; Mol. Wt: $283.31 ;{ }^{1} \mathrm{H}$ NMR (400 MHz, DMSO-d $\left.{ }_{6}\right) \delta /$ ppm: 7.41-7.60 (m, 5H, ArH), 7.69-7.79 (d, $\left.1 \mathrm{H}, J=8.1 \mathrm{~Hz}, J=8.7 \mathrm{~Hz}, 5^{\prime}-\mathrm{ArH}\right)$, 7.82-7.88 (d, 1H, $\left.J=8.2 \mathrm{~Hz}, 4^{\prime}-\mathrm{ArH}\right), 7.90-8.03$ (d, $\left.1 \mathrm{H}, J=8.2 \mathrm{~Hz}, 6^{\prime}-\mathrm{ArH}\right), 8.04-8.16$ (s, $\left.1 \mathrm{H}, 2^{\prime}-\mathrm{ArH}\right),{ }^{13} \mathrm{C}$ NMR (75 MHz, DMSO-d ${ }_{6}$ ) $/$ ppm: 122.8, 123.9, 128.7, 129.2, 130.1, 130.9, 133.5, 134.4, 148.4, 174.1; Anal. Calcd for $\mathrm{C}_{14} \mathrm{H}_{9} \mathrm{~N}_{3} \mathrm{O}_{2} \mathrm{~S}$ : C, 59.35; H, 3.20; N, 14.83. Found: C, 59.31; H, 3.18; N, 14.81.

4j. 2-phenyl-5-(p-tolyl)-1,3,4-thiadiazole

m.p. $155{ }^{\circ} \mathrm{C}, m / z$ : 252.07; Mol. Wt: 252.33 ; ${ }^{1} \mathrm{H}$ NMR (400 MHz, DMSO-d $)_{6} \delta / \mathrm{ppm}: 2.34\left(\mathrm{~s}, 3 \mathrm{H},-\mathrm{CH}_{3}\right)$, 7.41-7.60 (m, 5H, ArH), 7.69-7.80 (d, 2H, $J=7.9 \mathrm{~Hz}$, 3',5'-ArH), 7.90-7.98 (d, 2H, $\left.J=8.1 \mathrm{~Hz}, 2^{\prime}, 6^{\prime}-\mathrm{ArH}\right)$, ${ }^{13} \mathrm{C}$ NMR (75 MHz, DMSO-d ${ }_{6}$ ) $\delta /$ ppm: 21.3, 127.4, 128.7, 129.2, 129.5, 130.5, 130.9, 131.7, 133.5, 174.1; Anal. Calcd for $\mathrm{C}_{15} \mathrm{H}_{12} \mathrm{~N}_{2} \mathrm{~S}$ : C, 71.40; H, 4.79; N, 11.10. Found: C, 71.38; H, 4.77; N, 11.08.

4k. 2-(4-chlorophenyl)-5-(p-tolyl)-1,3,4-thiadiazole m.p. $160{ }^{\circ} \mathrm{C}, \mathrm{m} / z$ : 286.03; Mol. Wt: 286.78; ${ }^{1} \mathrm{H}$ NMR (400 MHz, DMSO-d $)_{6} \delta /$ ppm: $2.34\left(\mathrm{~s}, 3 \mathrm{H},-\mathrm{CH}_{3}\right)$, 7.29-7.38 (d, 2H, $\left.J=7.8 \mathrm{~Hz}, 3^{\prime \prime}, 5^{\prime \prime}-\mathrm{ArH}\right), 7.46-7.55$ (d, 2H, $\left.J=8.2 \mathrm{~Hz}, 3^{\prime}, 5^{\prime}-\mathrm{ArH}\right), 7.65-7.74$ (d, 2H, $J=$ $\left.7.8 \mathrm{~Hz}, 2^{\prime \prime}, 6^{\prime \prime}-\mathrm{ArH}\right), 8.02-8.15$ (d, 2H, $J=8.2 \mathrm{~Hz}, 2^{\prime}$, $\left.6^{\prime}-\mathrm{ArH}\right),{ }^{13} \mathrm{C}$ NMR $\left(75 \mathrm{MHz}, \mathrm{DMSO}-\mathrm{d}_{6}\right) \delta / \mathrm{ppm}^{\prime}$ 21.3, 127.4, 128.9, 129.3, 129.5, 130.5, 131.6, 131.7, 134.3, 174.1; Anal. Calcd for $\mathrm{C}_{15} \mathrm{H}_{11} \mathrm{ClN}_{2} \mathrm{~S}$ : C, 62.82; H, 3.87;
N, 9.77. Found: C, 62.80; H, 3.85; N, 9.75.

4l. 2-(3-chlorophenyl)-5-(p-tolyl)-1,3,4-thiadiazole m.p. $162{ }^{\circ} \mathrm{C}, m / z$ : 286.03; Mol. Wt: 286.78; ${ }^{1} \mathrm{H}$ NMR (400 MHz, DMSO-d 6 ) $\delta /$ ppm: $2.34\left(\mathrm{~s}, 3 \mathrm{H},-\mathrm{CH}_{3}\right)$, 7.29-7.38 (d, 2H, $\left.J=7.8 \mathrm{~Hz}, 3^{\prime \prime}, 5^{\prime \prime}-\mathrm{ArH}\right), 7.65-7.74$ (d, 2H, $\left.J=7.8 \mathrm{~Hz}, 2^{\prime \prime}, 6^{\prime \prime}-\mathrm{ArH}\right), 7.77-7.84$ (dd, $1 \mathrm{H}, J=$ $8.1 \mathrm{~Hz}, J=8.9 \mathrm{~Hz}, 5^{\prime}$-ArH), 7.86-7.89 (d, 1H, $J=8.1$ $\left.\mathrm{Hz}, 4^{\prime}-\mathrm{ArH}\right), 7.91-8.00$ (d, $\left.1 \mathrm{H}, J=8.3 \mathrm{~Hz}, 6^{\prime}-\mathrm{ArH}\right)$, 8.04-8.13 (s, 1H, 2'-ArH), ${ }^{13} \mathrm{C}$ NMR (75 MHz, DMSO$\left.\mathrm{d}_{6}\right) \delta / \mathrm{ppm}: 21.3,127.4,128.8,129.0,129.5,130.5$, 131.7, 134.8, 134.9, 174.1; Anal. Calcd for $\mathrm{C}_{15} \mathrm{H}_{11} \mathrm{ClN}_{2} \mathrm{~S}: \mathrm{C}, 62.82 ; \mathrm{H}, 3.87 ; \mathrm{N}, 9.77$. Found: C, $62.80 ; \mathrm{H}, 3.85 ; \mathrm{N}, 9.75$.

4m. 2-(2-chlorophenyl)-5-(p-tolyl)-1,3,4-thiadiazole m.p. $168{ }^{\circ} \mathrm{C}, m / z$ : 286.03; Mol. Wt: $286.78 ;{ }^{1} \mathrm{H}$ NMR (400 MHz, DMSO-d ${ }_{6}$ ) $\delta / \mathrm{ppm}: 2.34\left(\mathrm{~s}, 3 \mathrm{H},-\mathrm{CH}_{3}\right)$, 7.29-7.38 (d, 2H, $\left.J=7.8 \mathrm{~Hz}, 3^{\prime \prime}, 5^{\prime \prime}-\mathrm{ArH}\right), 7.41-7.60$ (m, 7H, ArH, 4', 5'-ArH), 7.65-7.74 (d, 2H, $J=7.8 \mathrm{~Hz}$, $\left.2^{\prime \prime}, 6^{\prime \prime}-\mathrm{ArH}\right), 7.75-7.84$ (d, 1H, $\left.J=8.2 \mathrm{~Hz}, 3^{\prime}-\mathrm{ArH}\right)$, 7.86-7.98 (d, $\left.1 \mathrm{H}, J=8.2 \mathrm{~Hz}, 66^{\prime}-\mathrm{ArH}\right),{ }^{13} \mathrm{C}$ NMR $(75$ MHz, DMSO-d 6 ) $\delta /$ ppm: 21.3, 127.3, 127.4, 128.9, 129.3, 129.5, 130.1, 130.5, 131.7, 132.2, 136.9, 174.1; Anal. Calcd for $\mathrm{C}_{15} \mathrm{H}_{11} \mathrm{ClN}_{2} \mathrm{~S}: \mathrm{C}, 62.82 ; \mathrm{H}, 3.87$; N, 9.77. Found: C, 62.80; H, 3.85; N, 9.75.

4n. 2-(4-methoxyphenyl)-5-(p-tolyl)-1,3,4-thiadiazole m.p. $220{ }^{\circ} \mathrm{C}, m / z$ : 282.08; Mol. Wt: 282.36; ${ }^{1} \mathrm{H}$ NMR (400 MHz, DMSO-d 6 ) $\delta /$ ppm: 2.34 (s, 3H, $-\mathrm{CH}_{3}$ ), 3.83 (s, 3H, $\left.-\mathrm{OCH}_{3}\right), 7.29-7.38\left(\mathrm{~d}, 2 \mathrm{H}, J=7.8 \mathrm{~Hz}, 3^{\prime \prime}, 5^{\prime \prime}-\right.$ ArH), 7.58-7.69 (d, 2H, $\left.J=8.1 \mathrm{~Hz}, 3^{\prime}, 5^{\prime}-\mathrm{ArH}\right)$, 7.65-7.74 (d, 2H, $\left.J=7.8 \mathrm{~Hz}, 2^{\prime \prime}, 6^{\prime \prime}-\mathrm{ArH}\right), 8.05-8.13$ (d, $\left.2 \mathrm{H}, J=8.4 \mathrm{~Hz}, 2^{\prime}, 66^{\prime}-\mathrm{ArH}\right),{ }^{13} \mathrm{C}$ NMR $(75 \mathrm{MHz}$, DMSO-d ${ }_{6}$ ) $\delta /$ ppm: $21.3,55.8,114.8,125.8,127.4$, 128.5, 129.5, 130.5, 131.7, 160.6, 174.1; Anal. Calcd for $\mathrm{C}_{16} \mathrm{H}_{14} \mathrm{~N}_{2} \mathrm{OS}$ : C, 68.06; H, 5.00; N, 9.92. Found: $\mathrm{C}$, $68.03 ; \mathrm{H}, 4.98 ; \mathrm{N}, 9.89$.

\section{RESULTS AND DISCUSSION}

In order to work out the envisaged protocol, a key reaction was conducted with thioacylhydrazone $\mathbf{3}(\mathbf{a}-\mathbf{n})$ in MeCN containing $2 \mathrm{~mol} \%$ of eosin Y under an air atmosphere (without air bubbling) by irradiation with visible light (green light-emitting diodes (LEDs), $\lambda$ $\max =535 \mathrm{~nm})$ at $\mathrm{rt}$. The reaction delivered the desired 2,5-disubtituted 1,3,4-thiadiazole $\mathbf{4}(\mathbf{a}-\mathbf{n})$ in $12 \%$ isolated yield after $24 \mathrm{~h}$ (Table 1 , entry 1). Following this experiment, a series of control experiments were performed, which indicates that an organic base is essential to give the desired product with high yield (97\%) (Table 1, entry 2) and TMP was found to be the best base (Table 2, entry 2 versus $6,7,9$ ). There was no product formation or it was formed in traces in the absence $(-)$ of any one of the reagents/catalyst (Table 1, entries 
Table 1. Screening and control experiments ${ }^{\mathrm{a}}$<smiles></smiles>

\begin{tabular}{|c|c|c|c|c|c|}
\hline Entry & Visible Light & Eosin $Y$ & Air & Time / h & Yield $/ \%^{b}$ \\
\hline 1 & + & + & + & 24 & $12^{\mathrm{c}}$ \\
\hline 2 & + & + & + & 10 & 97 \\
\hline 3 & - & + & + & 24 & n.r. ${ }^{\mathrm{d}}$ \\
\hline 4 & + & - & + & 24 & n.r. \\
\hline 5 & + & + & - & 24 & n.r. \\
\hline 6 & + & + & + & 10 & $45^{\mathrm{e}}$ \\
\hline 7 & + & + & $\mathrm{N}_{2}$ & 24 & trace \\
\hline 8 & + & + & $\mathrm{O}_{2}$ & 10 & 97 \\
\hline 9 & + & + & + & 10 & $97^{\mathrm{f}}$ \\
\hline 10 & + & + & + & 10 & $60^{\mathrm{g}}$ \\
\hline 11 & + & + & + & 10 & $42^{\mathrm{h}}$ \\
\hline
\end{tabular}

${ }^{\mathrm{a}}$ Reaction conditions: thioacylhydrazone (1.0 mmol), eosin Y (2.0 mol\%), TMP (2.0 equiv.), MeCN (3.0 mL), green LEDs 2.4W, $120 \mathrm{~lm}$ irradiation under an air atmosphere at rt.

${ }^{\mathrm{b}}$ Isolated yield of the product $(4 \mathrm{a}-\mathrm{n})$. n.r. $=$ no reaction.

${ }^{\mathrm{c}} \mathrm{The}$ reaction was conducted without TMP base in MeCN.

${ }^{\mathrm{d}}$ The reaction was carried out in the dark.

${ }^{\mathrm{e}}$ The reaction was carried out using $20 \mathrm{~W}$ CFL (compact fluorescent lamp).

${ }^{\mathrm{f}}$ The reaction was carried out with 3.0 equiv. of TMP.

${ }^{\mathrm{g}}$ The reaction was carried out 1.0 equiv. of TMP.

${ }^{\mathrm{h}}$ The reaction was carried out with $1.0 \mathrm{~mol} \%$ of eosin Y.

Table 2. Optimization of reaction conditions $\mathrm{s}^{\mathrm{a}}$<smiles>[R]/C=N/NC([R])=S</smiles>

3 (a-n) $\operatorname{eosin} \mathrm{Y}(\mathrm{mol} \%)$, base, air solvent, green LEDs, 10-16 h,rt<smiles>[R]c1nnc([As])s1</smiles>

$4(a-n)$

\begin{tabular}{|c|c|c|c|c|c|}
\hline Entry & Eosin $\mathrm{Y} / \mathrm{mol} \%$ & Base & Solvent $^{\mathrm{c}}$ & Time / h & Yield / \% ${ }^{b}$ \\
\hline 1 & 3 & TMP & $\mathrm{MeCN}$ & 10 & 97 \\
\hline 2 & 2 & TMP & $\mathrm{MeCN}$ & 10 & 97 \\
\hline 3 & 1 & TMP & $\mathrm{MeCN}$ & 10 & 42 \\
\hline 4 & 2 & TMP & $\mathrm{MeOH}$ & 16 & 72 \\
\hline 5 & 2 & TMP & $\mathrm{EtOH}$ & 16 & 62 \\
\hline 6 & 2 & DBU & $\mathrm{MeCN}$ & 16 & 52 \\
\hline 7 & 2 & DABCO & $\mathrm{MeCN}$ & 16 & 55 \\
\hline 8 & 2 & TMP & DMSO & 10 & 82 \\
\hline 9 & 2 & $\mathrm{Et}_{3} \mathrm{~N}$ & $\mathrm{MeCN}$ & 16 & 65 \\
\hline
\end{tabular}

${ }^{a}$ Reaction conditions: thioacylhydrazone (1.0 mmol), eosin Y (2.0 mol\%), TMP (2.0 equiv.), MeCN (3.0 mL), green LEDs 2.4W, $120 \mathrm{~lm}$ irradiation under an air atmosphere at rt.

${ }^{\mathrm{b}}$ Isolated yield of the product $(4 a-n)$. 


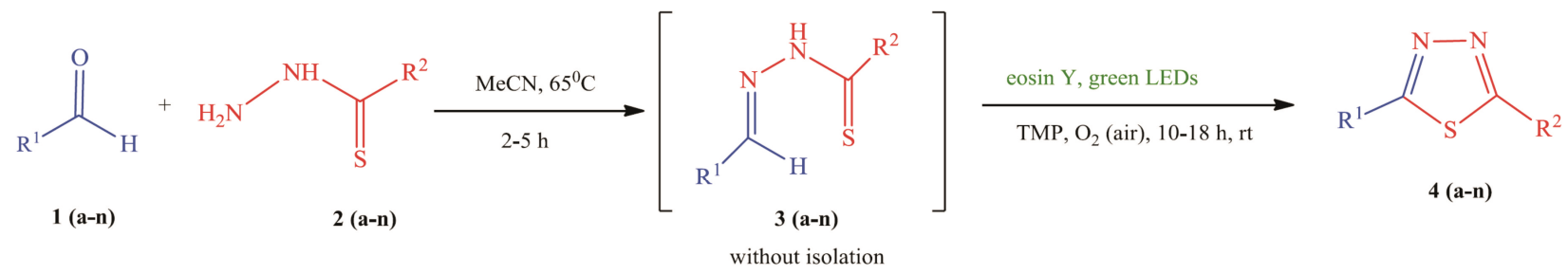

Scheme 2. One-pot facile synthesis of 1,3,4-thiadizole directly from aldehyde and thioacylhydrazide.

$3-5)$. The reaction did not proceed satisfactorily when a household $20 \mathrm{~W}$ fluorescent lamp was used instead of green LEDs (Table 1, entries 6 versus 2). Notably, the same result was obtained on using $\mathrm{O}_{2}$ (balloon) instead of an air atmosphere (Table 1, entry 8 versus 2), whereas in the absence of any gas or under a nitrogen atmo-sphere no product formation was detected (Table 1 , entry 5,7$)$. These results establish that visible light, base, photocatalyst and air all are essential $(+)$ for the reaction and support the photocatalytic model of the reaction. Thiadiaazole exhibit various biological activities and has greater synthetic utility in medicinal chemistry. The use of novel one-pot visible light irradiated synthesis using eosin $\mathrm{Y}$ as an organophotoredox catalyst fullfill the basic need of green chemistry.

Next, the reaction conditions were optimized with respect to solvents and the catalyst used in the reaction. In all the tested solvents (MeCN, DMSO, $\mathrm{MeOH}$ and EtOH) the yield of $4 a-n$ was $>55 \%$ (Table 2), which indicates that the reaction is not very sensitive to reac- tion media. MeCN was the best solvent in terms of the reaction time and yield (Table 2, entry 1), hence it was used throughout the synthesis. When the amount of the catalyst was decreased from $2 \mathrm{~mol} \%$ to $1 \mathrm{~mol} \%$, the yield of $\mathbf{4}(\mathbf{a}-\mathbf{n})$ considerably reduced (Table 2 , entry 3 ), but the use of $3 \mathrm{~mol} \%$ of the catalyst did not affect the yield (Table 2 , entry 1 ).

Under the established reaction conditions in hand, the reaction was tried in a one-pot procedure starting directly from an aldehyde $\mathbf{1}(\mathbf{a}-\mathbf{n})$ and an thioacylhydrazide $\mathbf{2}(\mathbf{a}-\mathbf{n})$ to give the desired product $\mathbf{4}(\mathbf{a}-\mathbf{n})$ as depicted in Scheme 2.

To our delight, it worked well and a number of symmetrical and unsymmetrical 2, 5-disubstituted 1,3,4thiadiazoles were successfully synthesized starting directly from various aldehydes $\mathbf{1}(\mathbf{a}-\mathbf{n})$ and thioacylhydrazides 2(a-n) (Tables 3$)$.

This clearly shows that the reaction is very mild and applicable to aryl and alkyl, tolerates considerable functional group variations like $\mathrm{MeO}, \mathrm{Br}, \mathrm{Cl}$ and $\mathrm{NO}_{2}$ in

Table 3. Eosin Y catalysed synthesis of 1,3,4-thiadizole

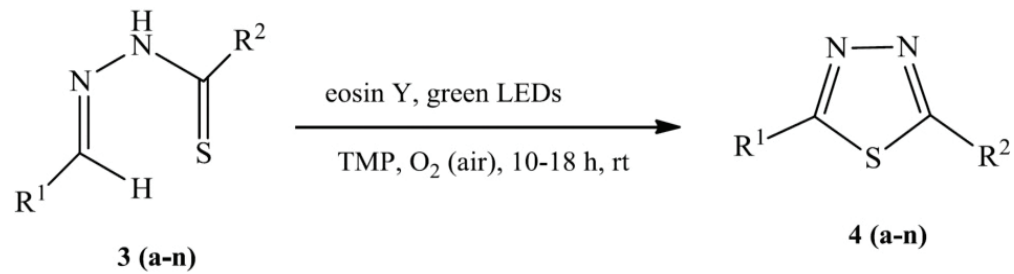

\begin{tabular}{|c|c|c|c|c|c|}
\hline Entry & $\mathrm{R}^{1}$ & $\mathrm{R}^{2}$ & Product & Time / h & Yield / \% \\
\hline 1 & 4-Cl. $\mathrm{C}_{6} \mathrm{H}_{4}$ & $\mathrm{C}_{6} \mathrm{H}_{5}$ & $4 a$ & 14 & 82 \\
\hline 2 & $3-\mathrm{Cl} . \mathrm{C}_{6} \mathrm{H}_{4}$ & $\mathrm{C}_{6} \mathrm{H}_{5}$ & $4 b$ & 14 & 85 \\
\hline 3 & 2-Cl. $\mathrm{C}_{6} \mathrm{H}_{4}$ & $\mathrm{C}_{6} \mathrm{H}_{5}$ & $4 \mathrm{c}$ & 13 & 87 \\
\hline 4 & $4-\mathrm{OCH}_{3} \cdot \mathrm{C}_{6} \mathrm{H}_{4}$ & $\mathrm{C}_{6} \mathrm{H}_{5}$ & $4 d$ & 10 & 96 \\
\hline 5 & $\mathrm{C}_{6} \mathrm{H}_{5}$ & $\mathrm{C}_{6} \mathrm{H}_{5}$ & $4 \mathrm{e}$ & 10 & 92 \\
\hline 6 & 4-Br. $\mathrm{C}_{6} \mathrm{H}_{4}$ & $\mathrm{C}_{6} \mathrm{H}_{5}$ & $4 \mathrm{f}$ & 14 & 85 \\
\hline 7 & $3-\mathrm{Br} \cdot \mathrm{C}_{6} \mathrm{H}_{4}$ & $\mathrm{C}_{6} \mathrm{H}_{5}$ & $4 g$ & 14 & 86 \\
\hline 8 & $4-\mathrm{O}_{2} \mathrm{NCC}_{6} \mathrm{H}_{4}$ & $\mathrm{C}_{6} \mathrm{H}_{5}$ & $4 \mathrm{~h}$ & 18 & 78 \\
\hline 9 & $3-\mathrm{O}_{2} \mathrm{~N}_{6} \mathrm{C}_{6} \mathrm{H}_{4}$ & $\mathrm{C}_{6} \mathrm{H}_{5}$ & $4 \mathrm{i}$ & 18 & 84 \\
\hline 10 & 4- $\mathrm{CH}_{3} \cdot \mathrm{C}_{6} \mathrm{H}_{4}$ & $\mathrm{C}_{6} \mathrm{H}_{5}$ & $4 j$ & 10 & 96 \\
\hline 11 & 4-Cl. $\mathrm{C}_{6} \mathrm{H}_{4}$ & $4-\mathrm{CH}_{3} \cdot \mathrm{C}_{6} \mathrm{H}_{4}$ & $4 \mathrm{k}$ & 12 & 83 \\
\hline 12 & $3-\mathrm{Cl} . \mathrm{C}_{6} \mathrm{H}_{4}$ & 4- $\mathrm{CH}_{3} \cdot \mathrm{C}_{6} \mathrm{H}_{4}$ & 41 & 12 & 86 \\
\hline 13 & 2-Cl. $\mathrm{C}_{6} \mathrm{H}_{4}$ & 4- $\mathrm{CH}_{3} \cdot \mathrm{C}_{6} \mathrm{H}_{4}$ & $4 \mathrm{~m}$ & 11 & 89 \\
\hline 14 & $4-\mathrm{OCH}_{3} \cdot \mathrm{C}_{6} \mathrm{H}_{4}$ & $4-\mathrm{CH}_{3} \cdot \mathrm{C}_{6} \mathrm{H}_{4}$ & $4 n$ & 10 & 97 \\
\hline
\end{tabular}




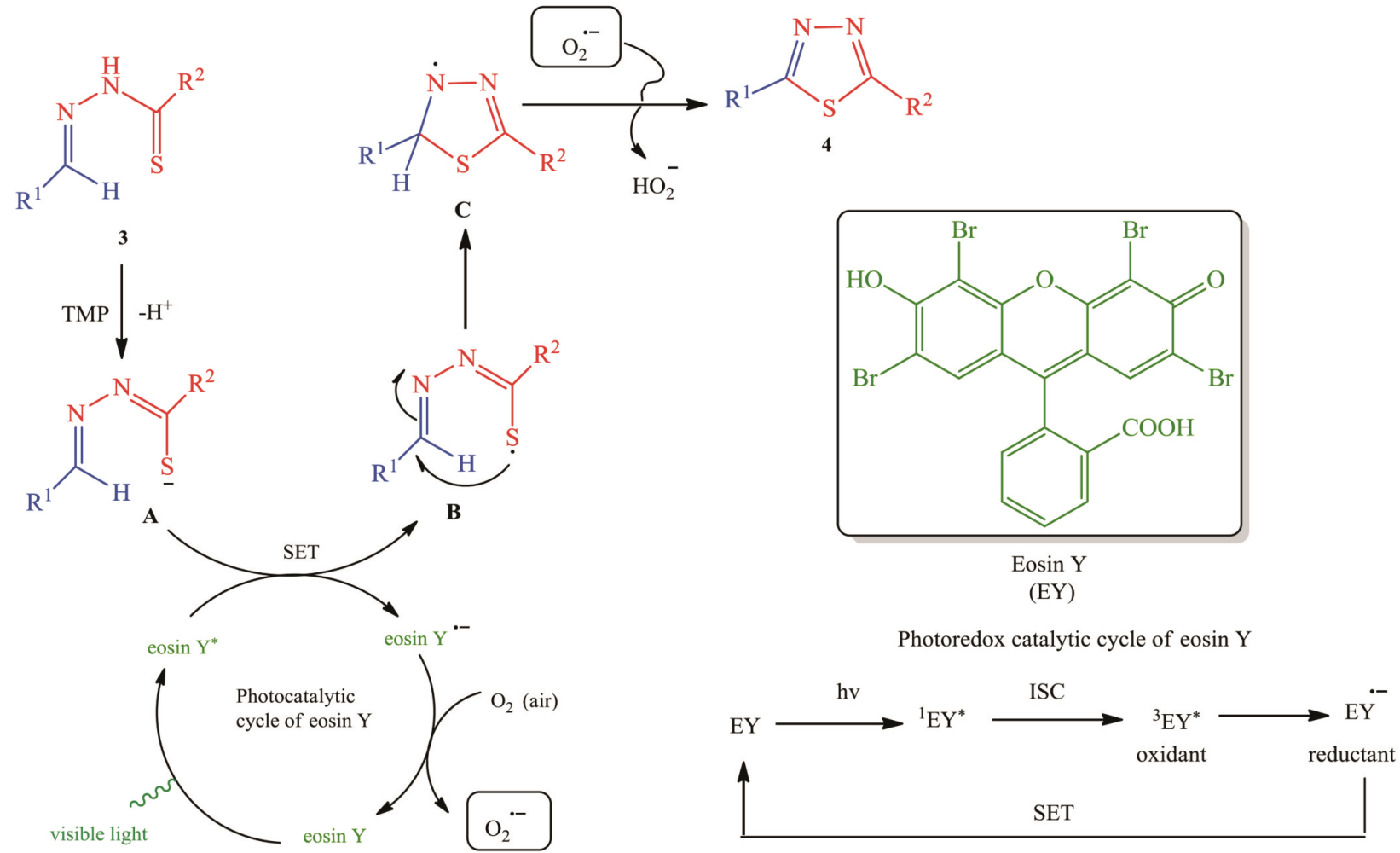

Scheme 3. Proposed mechanism for the visible light irradiated synthesis of 1,3,4-thiadiazole using eosin Y as photocatalyst.

the substrate, which results the desired product $\mathbf{4}(\mathbf{a}-\mathbf{n})$ in good to excellent yields (78-97\%). However, aldehydes $\mathbf{1}(\mathbf{a}-\mathbf{n})$ and thioacylhydrazides $\mathbf{2}(\mathbf{a}-\mathbf{n})$ with an electron-donating group on the aromatic ring appear to react faster and afford marginally higher yields in comparison to those bearing an electron withdrawing group.

On the basis of the above observations and the literature precedents, a plausible mechanism involving photoredox catalysis for the oxidative cyclization of acylhydrazones is depicted in Scheme 3. On absorption of visible light, the organophotoredox catalyst eosin Y (EY) is excited to its singlet state ${ }^{1} \mathrm{EY}^{*}$ which through inter system crossing (ISC) comes to its more stable triplet state ${ }^{3} \mathrm{EY}^{*}$ and undergoes a single electron transfer (SET). ${ }^{3} \mathrm{EY}^{*}$ may undergo both reductive and oxidative quenching. ${ }^{45-49}$ A SET from A to ${ }^{3} \mathrm{EY}^{*}$ generates thioacyl radical $\mathrm{B}$, which undergoes intramolecular cyclization (5-endo-trig) to form $\mathrm{C}$ followed by attack of $\mathrm{O}_{2}^{--}$to give the product 4 , successively. The formation of superoxide radical anion $\left(\mathrm{O}_{2}^{--}\right)$during the reaction was confirmed by the detection of the resulting $\mathrm{H}_{2} \mathrm{O}_{2}$ using $\mathrm{KI} /$ starch indicator. $^{50}$

\section{CONCLUSION}

In conclusion, we have developed a novel organocatalysed method for the synthesis of 1,3,4-thiadiazoles directly from aldehydes and thioacylhydrazides in a one-pot procedure by using inexpensive eosin $\mathrm{Y}$ as a powerful organophotoredox catalyst at $\mathrm{rt}$. The reaction involves visible light, a base and $\mathrm{O}_{2}$ (air) as a valuable reagents. This synthetic pathway includes a superior visible light promoted and Eosin Y organophotoredox catalysed methodology, which is superior in comparison to all other alternative synthetic methods for 1,3,4-thiadiazoles. This synthesis widens the scope of substrates for visible light photoredox reactions. The present methodology also offers many advantages of green chemistry such as high atom economy, reduced reaction time, one-pot consolidated procedure and high efficiency.

Acknowledgements. We sincerely thank SAIF, CDRI, Lucknow and IISc Banglaore for providing microanalyses and spectra.

\section{REFERENCES}

1. N. S. Lewis, Science 315 (2007) 798-801.

2. O. Morton, Nature 443 (2006) 19-22.

3. D. G. Nocera, Daedalus 135 (2006) 112-115.

4. D. Mandler and I. Willner, J. Am. Chem. Soc. 106 (1984) 5352 5353.

5. O. Ishitani, S. Yanagida, S. Takamuku, and C. Pac, J. Org. Chem. 52 (1987) 2790-2796.

6. D. A. Nicewicz and T. M. Nguen, ACS Catal. 4 (2014) 355.

7. J. Xiea, H. Jina, P. Xua, and C. Zhu, Tetrahedron Lett. 55 (2014) 36.

8. X. Lang, X. Chen, and J. Zhao, Chem. Soc. Rev. 43 (2014) 473. 
9. J. Hu, J. Wang, T. H. Nguyen, and Zheng, Beilstein J. Org. Chem. 9 (2013) 1977.

10. D. Rovelli, M. Fagnoni, and A. Albini, Chem. Soc. Rev. 42 (2013) 97.

11. T. P. Yoon, M. A. Ischay, and J. N. Du, Nat. Chem. 2 (2010) 527.

12. D. A. Nicewicz and D. W. C. MacMillan, Science 322 (2008) 77.

13. M. A. Ischay, M. E. Anzovino, J. Du, and T. P. Yoon, J. Am. Chem. Soc. 130 (2008) 12886.

14. J. M. R. Narayanam and C. R. J. Stephenson, Chem. Soc. Rev. 40 (2011) 102.

15. J. M. R. Narayanam, J. W. Tucker, and C. R. J. Stephenson, J. Am. Chem. Soc. 131 (2009) 8756.

16. J. W. Tucker and C. R. J. Stephenson, J. Org. Chem. 77 (2012) 1617.

17. X.-J. Yang, B. Chen, L.-Q. Zheng, L.-Z. Wu, and C.-H. Tung, Green Chem. 16 (2014) 1082.

18. Y. C. Teo, Y. Pan and C. H. Tan, ChemCatChem. 5 (2013) 235.

19. K. Fidaly, C. Ceballos, A. Falguières, M. S.-I. Veitia, A. Guy, and C. Ferroud, Green. Chem. 14 (2012) 1293.

20. D.-T. Yang, Q.-Y. Meng, J.-J. Zhong, M. Xiang, Q. Liu, and L.Z. Wu, Eur. J. Org. Chem. (2013) 7528.

21. Y.-Q. Zou, J.-R. Chen, X.-P. Liu, L.-Q. Lu, R. L. Davis, K. A. Jørgensen and W.-J. Xiao, Angew. Chem. Int. Ed. 51 (2012) 784.

22. D. P. Hari and B. König, Org. Lett. 13 (2011) 3852.

23. M. Neumann, S. Füldner, B. König, and K. Zeitler, Angew. Chem. Int. Ed. 50 (2011) 951.

24. V. Rey, S. Soria-Catro, J. E. Argüello, and A. B. Peñéñory, Tetrahedron Lett. 50 (2009) 4720.

25. S. Schenone, C. Brullo, O. Bruno, F. Bondavalli, A. Ranise, W. Filippelli, B. Rinaldi, A. Capuano, and G. Falcone, Bioorg. Med. Chem. 14 (2006) 1698.

26. F. Clerici and D. Pocar, J. Med. Chem. 44 (2001) 931

27. M. R. Stillings, A. P. Wellbournt, and D. S. Walter, J. Med. Chem. 29 (1986) 2280.

28. C. B. Chapleo, M. Myers, P. L. Myers, J. F. Saville, A. C. B. Smith, M. R. Stillings, I. F.Tulloch, D. S. Walter, and A. P. Welbourn, J. Med. Chem. 29 (1986) 2273.

29. M. D. Mullicon, M. W. Willson, D. T. Conor, C. R. Kostlan, and D. J. Schrier, J. Med. Chem. 36 (1993) 1090.

30. S. Servi, M. Genc, S. Gur, and M. Koca, Eur. J. Med. Chem. 40 (2005) 687.
31. A. Foroumadia, S. Enamic, A. Hassanzadehb, M. Rajaeeb, K. Sokhanverb, M. Hasan, and A. Shafieea, Bioorg. Med. Chem. Lett. 15 (2005) 4488.

32. E. E. Oruc, S. Rollas, F. Kandemirli, N. Shvets, and A. S. Dimoglo, Bioorg. Med. Chem. Lett. 12 (2004) 5651.

33. J. Matysiak, Chem. Pharm. Bull. 54 (2006) 988.

34. E. Roduner, W. Kaim, B. Sarkar, V. B. Urlacher, J. Pleiss, R. Glser, W.-D. Einicke, G. A. Sprenger, U. Beifuß, E. Klemm, C. Liebner, H. Hieronymus, S.-F. Hsu, B. Plietker, and S. Laschat, ChemCatchem 5 (2013) 82.

35. Z. Shi, C. Zhang, C. Tang, and N. Jiao, Chem. Soc. Rev. 41 (2012) 3381

36. D. P. Hari, T. Hering, and B. König, Org. Lett. 14 (2012) 5334.

37. S. Samanta, S. Das, and P. Biswas, J. Org. Chem. 78 (2013) 11184.

38. G. Crank and A. J. Mursydi, PhotoChem. Photobio. 53 (1990) 30.

39. Y.-Q. Zou, J.-R. Chen, X.-P. Liu, L.-Q. Lu, R. L. Davis, K. A. Jørgensen, and W.-J. Xiao, Angew. Chem. Int. Ed. 51 (2012) 784.

40. J. Zhang, L. Wang, Q. Liu, Z. Yang, and Y. Huang, Chem. Commun. 49 (2013) 11662.

41. I. R. Siddiqui, P. K. Singh, V. Srivastava, and J. Singh, Indian J. Chem. 46B (2007) 1716-1720.

42. V. Srivastava, P. K. Singh, and P. P. Singh, Croat. Chem. Acto 87(2) (2014) 91.

43. V. Srivastava, P. K. Singh, and P. P. Singh, Chemistry of Heterocyclic Compounds 50(4) (2014) 573.

44. P. K. Singh and I. R. Siddiqui, Indian J. Chem. 48B (2009) 1013

45. D. C. Neckers and O. M. Valdes-Aguilera, Adv. Photochem. 18 (1993) 315.

46. M. V. Encinas, A. M. Rufs, S. G. Bertolotti, and C. M. Previtali, Polymer 50 (2009) 2762.

47. T. Lizarides, T. McCormick, P. Du, G. Luo, B. Lindley, and R. Eisenberg, J. Am. Chem. Soc. 131 (2009) 9192.

48. S. H. Lee, D. H. Nam, and C. B. Park, Adv. Synth. Catal. 351 (2009) 2589

49. T. Xiao, X. Dong, Y. Tang, and L. Zhou, Adv. Synth. Catal. 354 (2012) 3195 .

50. G. K. Fekarurhobo, S. S. Angaye, and F. G. Obomann, J. Emerg. Trends Engg. Appl. Sci. (JETEAS), 4 (2013) 394. 